\title{
Do health care providers discuss HIV with older female patients?
}

\author{
Rauf WN, BSc, MBBS, DipPEC(SA), MMed(Fam Med)UP \\ Department of Family Medicine, Kalafong Hospital and University of Pretoria, South Africa \\ Correspondence to: DrWN Rauf: e-mail: nisa.rauf@up.ac.za \\ Keywords: HIV; older females; older women; discussion; HIV talks; consultations
}

\begin{abstract}
SA Fam Pract 2010;52(6):573-575

Background: The World Health Organization expressed concern that older people in Africa who are ignored and excluded from HIV-prevention programmes are increasingly being infected with HIV. Studies show an inadequate awareness of the risks of HIV in the older female population. Older women are more susceptible to HIV because menopausal changes, such as vaginal dryness and thinning, increase the risk of HIV infection through tears and abrasions during unprotected sex. Furthermore, progression from HIV to AIDS occurs faster in the older population. Older women need to understand how HIV is transmitted and how it can be prevented in order to protect themselves. It may be postulated that health care providers are reluctant to discuss HIV-related issues with older female patients. The aim of this study was to determine whether older women could recall receiving HIV-related information from health care providers.
\end{abstract}

Methods: This was a prospective cross-sectional study among 100 women, aged 50 to 80 , who attended the out-patient section at the Tshwane District Hospital during November and December 2006. Trained research assistants collected data in the patients' mother tongue. A questionnaire was administered during face-to-face interviews.

Results: Only $36 \%$ (95\% confidence interval [Cl]: $26.6 \%$ to $45.4 \%$ ) of the respondents recalled receiving prior information regarding HIV from their health care providers. Of those, $19(52.78 \%)$ said it was in the form of voluntary counselling and testing (VCT), and the remaining 17 (47.22\%) received this information during general consultations. There was a statistically significant difference ( $p=0.003$; odds ratio [OR]: $0.26 ; 95 \% \mathrm{Cl}: 0.09-0.69)$ between their age stratification of 50 to 59 years and 60 to 80 years with respect to receiving information regarding HIV. In total, $23 \%$ of the respondents said they had been tested for HIV and most $(n=19 ; 82.61 \%)$ said they had received counselling. However, it is cause for concern that $17.39 \%$ $(n=4)$ of the respondents who had been tested could not recall receiving counselling.

Conclusions: This study shows that older women are neglected regarding the provision of HIV-related information. HIV and its risks to older patients is not routinely part of patient care. VCT seems to be an effective solution. However, there are problems even within the VCT system, since some of the participants claim to have been tested for HIV, without receiving counselling. It is suggested that part of the challenge may lie in the mindset of health care providers. Family physicians need to become proactive in terms of their own practice as well as in terms of their engagement with other health care professionals and students.

(P) Peer reviewed. (Submitted: 2010-03-08, Accepted: 2010-04-21). ๑ SAAFP

\section{Introduction}

The World Health Organization expressed concern that, while older people in Africa are increasingly infected with HIV, they are ignored and excluded from HIV-prevention programmes. $^{1}$ Studies show that there is inadequate awareness of the risks of HIV and a rapid increase in HIV infection in the older population. ${ }^{2-5}$ The 2005 South African National HIV Prevalence, HIV Incidence, Behaviour and Communication Survey reported a $5.8 \%$ prevalence of HIV in the population of people aged 50 years and older. ${ }^{6}$ UNAIDS estimated that 2.8 million people aged 50 years and older were living with HIV in South Africa in 2006 and the prevalence in the 50- to 54-year-old population was $10.8 \% .{ }^{1}$ Sixty-seven per cent of middle-aged and older women attending out-patient clinics in neighbouring Zimbabwe were found to be HIV positive. ${ }^{7}$

It is a reality that menopausal and postmenopausal women are more susceptible to HIV than younger women. Physically, changes during menopause, such as vaginal dryness and thinning, increase their risk of HIV infection through tears and abrasions during unprotected sex. Moreover, the progression from HIV to AIDS is faster in the older population. ${ }^{5}$ Socially, their risk of HIV exposure is increased because of multiple partnering, especially of older men with younger girlfriends and their own older wives. ${ }^{8,9}$ 
It may be postulated that the focus of HIV-awareness campaigns on the youth and young adults leaves older adult women without knowledge of their own vulnerability to HIV infection. Older women need to understand how HIV is spread in order to protect themselves.

To gain insight into the problem of the visibility of older women to health care providers, a study of older women attending the out-patient section of the Tshwane District Hospital (TDH) was undertaken. The aim of the study was to investigate whether the women could recall receiving HIV-related information from health care providers. The objective was to determine the percentage of participants who reported receiving HIV-related information from health care providers and whether they received this information during general consultations or in the form of HIV voluntary counselling and testing (VCT).

\section{Methodology}

Data were prospectively collected during November and December 2006 through systematic sampling of women aged 50 to 80 years who attended the out-patients section of TDH on Monday to Friday of each week. This hospital provides primary health care services to patient living in the central and eastern suburbs of Tshwane. The administration clerk of the out-patient section compiled a list of all women in the requisite age group who attended at the time of sampling. Every third patient was invited to participate in the study and those who were willing were included until a sample of 100 was obtained. The sample size was calculated by nQuery version 4 . This sample size was required to obtain a two-sided $95 \%$ confidence interval $(\mathrm{Cl})$ for the proportion of women who received HIVrelated information from health care providers. Patients with mental incompetence were excluded from the study. Data were collected by trained research assistants in the mother tongue of the patients. The assistants administered the questionnaire during face-to-face interviews with the patients. The validity of the results was increased through the interviewers being able to clarify questions and answers. The questionnaire was designed by reviewing previous studies and was finalised by a team of family physicians working in this field. The questionnaire was piloted with 20 women.

Data were analysed through univariate statistical analysis. The Intercooled Stata 8.1 (Intercooled for Windows; STATA Corp, College Station, Tex; 2003) statistical package was used. The proportion of women who received information from health care providers was estimated along with a $95 \% \mathrm{Cl}$. Chi-square testing was used for the data analysis to determine any significant differences for different demographic groups. Testing was at the 0.05 level of significance. Odds ratios (OR) were also calculated.

The study was approved by the University of Pretoria's Research Ethics Committee. All the requirements of this committee were fulfilled, including those with regard to informed consent, anonymity and confidentiality. Patients were interviewed during the waiting period before seeing a doctor to avoid delaying them due to this research project. There was no risk or direct benefit involved.

\section{Results}

Of the 100 patients studied, $57 \%$ were between 50 and 59 years of age and $43 \%$ were aged between 60 and 80 years. The majority of respondents were black $(59 \%)$, with the remainder being predominantly white $(36 \%)$, while $5 \%$ were from other racial groups.

Only $36 \%$ (95\% Cl; $26.6 \%$ to $45.4 \%$ ) of the respondents recalled receiving prior information regarding HIV from their health care providers (Figure 1). Of those, 19 (52.78\%) said it was in the form of VCT, and the remaining 17 (47.22\%) received this information during general consultations (Figure 2). There was a statistically significant difference ( $p=0.003$; OR: 0.26 ; 95\% Cl: 0.09-0.69) between their age stratification of 50 to 59 years and 60 to 80 years with respect to receiving information regarding HIV. It proved that 60- to 80-year-old women were more neglected than 50 - to 59-year-old women regarding the provision of HIVrelated information. No statistically significant difference ( $p=0.152$; OR: $1.91 ; 95 \% \mathrm{Cl}: 0.72-5.25$ ) between black and white racial groups was observed in terms of receiving HIV-related information.

Of the 100 patients studied, less than a quarter $(n=23)$ said they had been tested for HIV. Most of those patients $(n=19$; $82.61 \%$ ) said they had received counselling. However, it is cause for concern that $17.39 \%(n=4)$ of the respondents who had been tested could not recall receiving counselling.

Figure 1: Participants who recalled receiving HIV-related information from health care providers

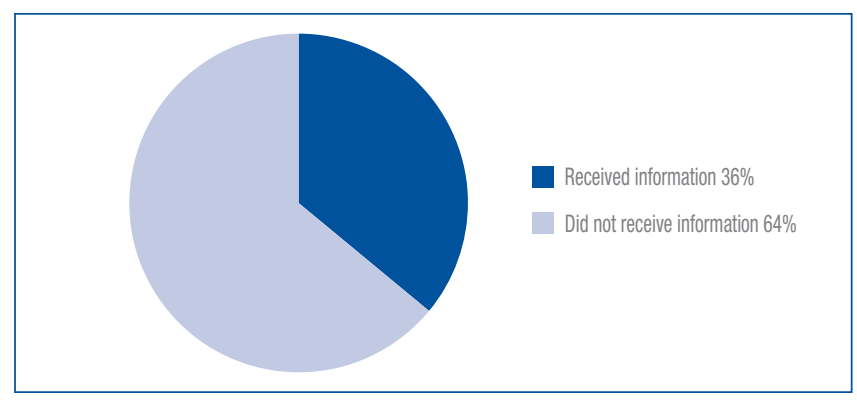

Figure 2: The proportion of patients who reported receiving HIV-related information during VCT versus general consultations

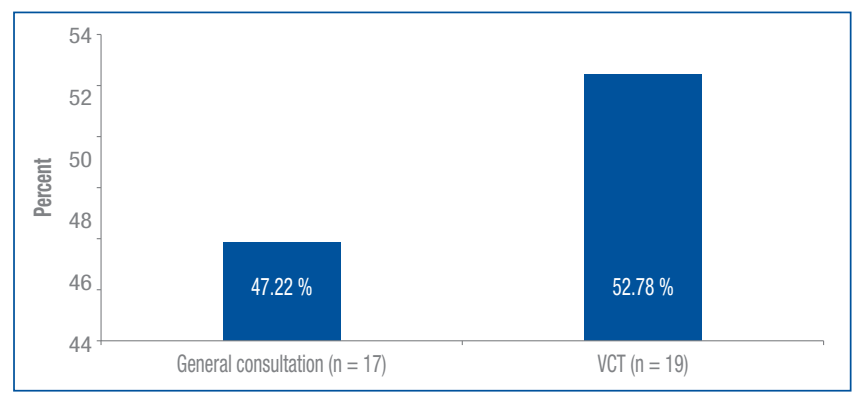




\section{Discussion}

The results of this study suggest that health care providers do not communicate routinely about HIV with older female patients during consultations. This is in keeping with local and international observations ${ }^{8-10}$ where, for example, a US study found that only $38 \%$ of older women report receiving such information from their health care providers. ${ }^{4}$

While the literature indicates that older women want to talk about HIV, it is the health care providers who are reluctant to discuss this issue with them. ${ }^{10,11}$ Most primary care physicians, for example, do not discuss HIV risk factors with patients aged 50 years and older. ${ }^{10}$ Aside from the pressures of time constraints, shortage of medical personnel and long queues of patients, there is the problem of health care providers' mindset. ${ }^{10,11}$ Health care providers generally find it difficult to bring up the subject of HIV and related sexual issues with older patients. Many of them fear that they may offend their patients by introducing the topic without the patients' prior enquiry. ${ }^{10-12}$

Once the issue of HIV risk is raised, VCT seems to be an effective solution. VCT for HIV is an important step in improving awareness of HIV prevention and treatment. ${ }^{13,14}$ However, a South African baseline review of services demonstrates low levels of VCT. ${ }^{13}$ From this study, it would seem that there are problems even within the system, since some of the participants claim to have been tested for HIV without receiving counselling.

Given the risks of HIV infection to older women, there is a need for health care providers to become more proactive in the delivery of routine counselling services. Combined efforts at multiple levels are required. Primary health care doctors and nurses can potentially be involved in improving the service delivered during consultations. Among other things, physicians can make HIV a routine aspect of consultation and care for older patients, especially women. Family physicians can initiate small-group discussions with nurses and students to make them aware of HIV in older patients and to train them on how to initiate discussions of HIV risk and sexuality in older people. Family physicians can also help to improve the present VCT system.

\section{Limitations}

This study was limited to the patients attending the outpatient section of TDH, with most of the patients living in Pretoria and surrounds. The results of the study could, therefore, not be extrapolated to the general population. This study questionnaire was limited to older patients and did not determine health care providers' perspectives regarding this issue. Future studies can be undertaken to investigate why health care providers do not routinely discuss HIV-related issues with older women.

\section{Conclusion and suggestions}

This study shows that older women are neglected regarding the provision of HIV-related information. HIV and its risks to older patients are not routinely part of patient care. VCT seems to be an effective solution, although, as mentioned in the article, there are also problems within the VCT system. It is suggested that part of the challenge may lie in the mindset of health care providers. As a way forward, family physicians need to become proactive on the issue, both in terms of their own practice and in terms of their engagement with other health care professionals and students.

\section{Acknowledgement}

I acknowledge the facilities and support provided by the Department of Family Medicine at the University of Pretoria to carry out this project. Guidance provided by Dr S Hitchcock during this study is highly appreciated. I am thankful to Prof Tessa Marcus and other colleagues in the department for their valuable comments on this manuscript.

\section{References}

1. World Health Organization. Impact of HIV/AIDS on older people in Africa Available from http://www.who.int/ageing/projects/HIV/en (Accessed 10/02/2010).

2. Yates ME, Stellato RK, Johannes CB, Avis NE. The importance of AIDSrelated knowledge for mid-life and older women. AIDS Educ Prev 1999 Jun;11(3):224-31.

3. McCalman CL. Barriers and motivators for low-income Brazilian women in metropolitan Belo Horizonte: Insights for AIDS prevention. Health Care Women Int 2003 Jul;24(6):565-85.

4. Henderson SJ, Bernstein LB, George DM, Doyle JP, Paranjape AS, CorbieSmith G. Older women and HIV: How much do they know and where are they getting their information? J Am Geriatr Soc 2004 Sep;52(9):1549-53.

5. Schable B, Chu SY, Diaz T. Characteristics of women 50 years of age or older with heterosexually acquired AIDS. Am J Public Health 1996 Nov; 86(11):1616-8

6. South African National HIV Prevalence, HIV Incidence, Behaviour and Communication Survey. Cape Town: Human Sciences Research Council; 2005. Available from http://0-www.hst.org.za.innopac.up.ac.za/indicators/ HIV_AIDS/HIV_HouseholdSurvey2005.pdf (Accessed 20/11/2008).

7. Mbizvo EM, Msuya SE, Hussain A, Chirenje MZ, Stray-Pedersen B. HIV prevalence in Zimbabwean women: 54-67\% knowledge and perceived risk. Int J STD AIDS 2003 Mar;14(3):202-7.

8. Maharaj P, Cleland J. Risk perception and condom use among married or cohabiting couples in KwaZulu-Natal, South Africa. Int Fam Plann Perspect 2005 Mar;31(1):24-9.

9. Fredlund VG, Hartung TK, Nash J, Ngubane N. AIDS awareness and sexual behaviour in a high HIV prevalence area in rural northern KwaZulu-Natal, South Africa. Inter J STD AIDS 2002 Dec; 13(12):829-32.

10. Gerbert B, Maguire BT, Coates TJ. Are patients talking to their physicians about AIDS? Am J Pub Heath 1990 Apr; 80(4):467-8.

11. Marks G, Richardson JL, Crepaz N, et al. Are HIV care providers talking with patients about safer sex and disclosure? A multi-clinic assessment. AIDS 2002;16(14):1953-7.

12. Mabunda G. HIV knowledge and practices among rural South Africans. J of Nur Scholarship 2004;36(4):300-4.

13. Pronyk PM, Kim JC, Makhubele MB, Hargreaves JR, Mohlala R, Hausler HP. Introduction of voluntary counseling and rapid testing for HIV in rural South Africa: From theory to practice. AIDS Care 2002 Dec;14(6):859-65.

14. Rohleder P, Swartz L. What I've noticed what they need is the stats: Lay HIV counsellors' reports of working in a task-oriented health care system. AIDS Care 2005 Apr;17(3):397-406. 